\title{
Porous colloidal hydrogels formed by coordination-driven self- assembly of charged metal-organic polyhedra
}

\author{
Zaoming Wang ${ }^{[a, b]}$ Gavin A. Craig, ${ }^{[a]}$ Alexandre Legrand, ${ }^{[a]}$ Frederik Haase,${ }^{[a]}$ Saori Minami, ${ }^{[c]}$ Kenji \\ Urayama, ${ }^{[\mathrm{c}]}$ and Shuhei Furukawa ${ }^{*[a, b]}$
}

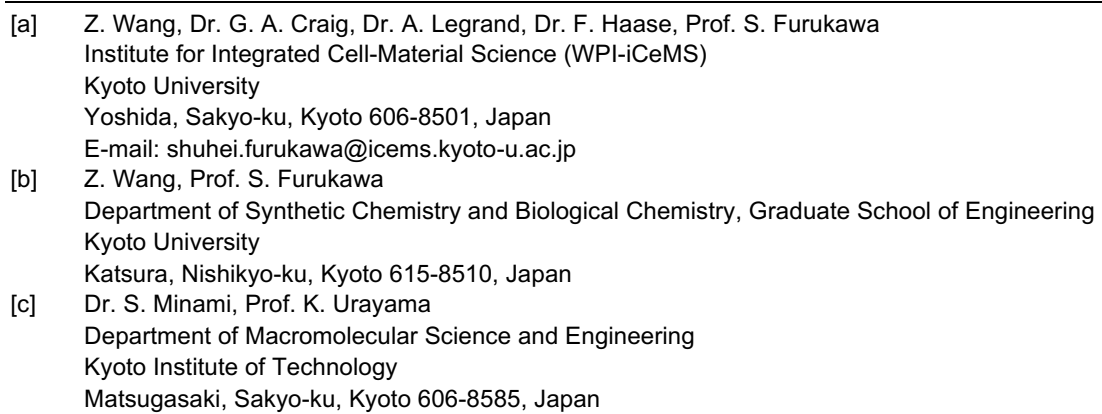

Supporting information for this article is given via a link at the end of the document.

\begin{abstract}
Introduction of porosity into supramolecular gels endows soft materials with functionalities for molecular encapsulation, release, separation and conversion. Metal-organic polyhedra (MOPs), discrete coordination cages containing an internal cavity, have recently been employed as building blocks to construct polymeric gel networks with potential porosity. However, most of the materials can only be synthesized in organic solvents, and the examples of porous, MOPbased hydrogels are scarce. Here, we demonstrate the fabrication of porous hydrogels based on $\left[\mathrm{Rh}_{2}(\mathrm{OH} \text {-bdc })_{2}\right]_{12}$, a rhodium-based MOP containing hydroxyl groups on its periphery $(\mathrm{OH}-\mathrm{bdc}=5$-hydroxy-1,3benzenedicarboxylate). By simply deprotonating $\left[\mathrm{Rh}_{2}(\mathrm{OH}-\mathrm{bdc})_{2}\right]_{12}$ with the base $\mathrm{NaOH}$, the supramolecular polymerization between MOPs and organic linkers can be induced in the aqueous solution, leading to the kinetically controllable formation of hydrogels with hierarchical colloidal networks. When heating the deprotonated MOP, $\mathrm{Na}_{\times}\left[\mathrm{Rh}_{24}(\mathrm{O}-\mathrm{bdc})_{\times}(\mathrm{OH}-\mathrm{bdc})_{24-x}\right]$, to induce gelation, the MOP was found to partially decompose, affecting the mechanical property of the resulting gels. By applying a post-synthetic deprotonation strategy, we show that the deprotonation degree of the MOP can be altered after the gel formation without serious decomposition of the MOPs. Gas sorption measurements confirmed the permanent porosity of the corresponding aerogels obtained from these MOP-based hydrogels, showing potentials for applications in gas sorption and catalysis.
\end{abstract}

\section{Introduction}

Supramolecular gels are fascinating soft materials formed by hierarchical self-assembly of low molecular weight gelators (LMWGs). ${ }^{[1]}$ Utilizing noncovalent interaction such as hydrogen bonding, metal-ligand coordination, hydrophobic or electrostatic interactions, well designed LMWGs self-assemble to form supramolecular networks, which show unique properties for applications in separation, drug-delivery, tissue engineering, sensing and optoelectronic devices. ${ }^{[2]}$ Among the efforts to tune the gel structures and properties, endowing supramolecular gels with predefined cavities is expected to imbue these soft materials with additional functionalities based on their potential host-guest chemistry. ${ }^{[3]}$ In particular, hydrogels formed from the cavitycontaining LMWGs are of special interest because they offer great promise in biological and environmental applications such as drug delivery and pollutants removal, which can make full use of the cavity-based host-guest chemistry. ${ }^{[4]}$ Most examples of cavitycontaining LMWGs reported so far are limited to traditional organic macrocycles, such as cucurbit[n]urils, ${ }^{[5]}$ cyclodextrins ${ }^{[6]}$, calix[n]arenes ${ }^{[7]}$ and pillar[n]arenes. ${ }^{[8]}$ Even though these macrocycles provide a well-defined host environment for guest molecules inside hydrogels, chemical synthesis of water-soluble macrocycles with tunable cavity sizes, shapes and surface functionality are still challenging. ${ }^{[9]}$

Coordination-driven self-assembly can be used to prepare cavity-containing molecules with well-defined sizes and shapes. ${ }^{[10]}$ Among them, metal-organic cages (MOCs) or metalorganic polyhedra (MOPs), a class of coordination cages with well-defined internal cavities, are potential precursors to incorporate cavities into hydrogels. ${ }^{[11]}$ By designing reactive binding sites on the periphery of cages, MOPs have been employed as building blocks to assemble or crosslink with other molecular units into $2 \mathrm{D}$ or $3 \mathrm{D}$ extended frameworks. ${ }^{[12]}$ Specifically, by reacting with polymer linkers, MOPs have been integrated into supramolecular gels with diverse properties, including guest encapsulation, self-healing and stimuli sensitivity. ${ }^{[13]}$ Recently, we reported the fabrication of supramolecular gels based on rhodium-based cuboctahedral MOPs, $\left[\mathrm{Rh}_{2}\left(\mathrm{C}_{12} \text {-bdc }\right)_{2}\right]_{12} \quad\left(\mathrm{C}_{12} \mathrm{RhMOP} ; \quad \mathrm{C}_{12}\right.$-bdc $=5$ dodecoxybenzene-1,3-dicarboxylate) or $\left[\mathrm{Rh}_{2}(\mathrm{bdc})_{2}\right]_{12}$ (HRhMOP; bdc = benzene-1,3-dicarboxylate) with shorter linker, 1,4bis(imidazol-1-ylmethyl)benzene (bix), by controlling the selfassembly pathway. ${ }^{[14]}$ Compared to macrocyclic molecules, the size, shape and chemical functionality of MOPs can be easily tuned by ligand design with easily accessible molecules, leading to supramolecular gels with varied host-guest chemistry. ${ }^{[15]}$ However, only a few hydrogels assembled from MOPs are reported to date ${ }^{[16]}$ most likely because most reported MOPs are 
(a)

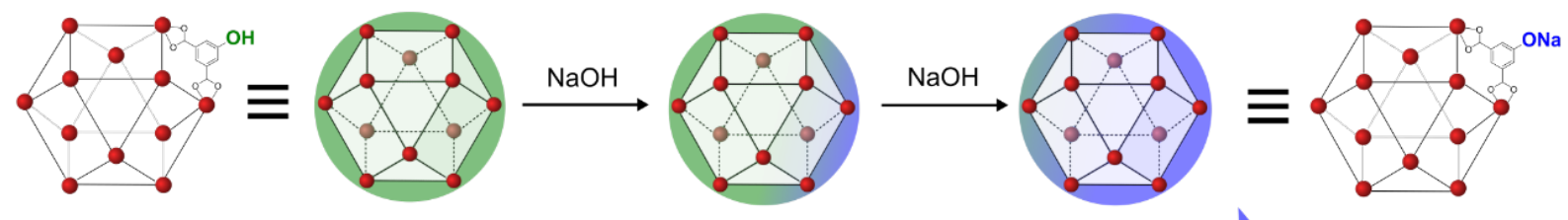

$\left[\mathrm{Rh}_{24}(\mathrm{OH}-\mathrm{bdc})_{24}\right]$ Increasing deprotonation degree of MOP

$\mathrm{Na}_{24}\left[\mathrm{Rh}_{24}(\mathrm{O}-\mathrm{bdc})_{24}\right]$

(b)
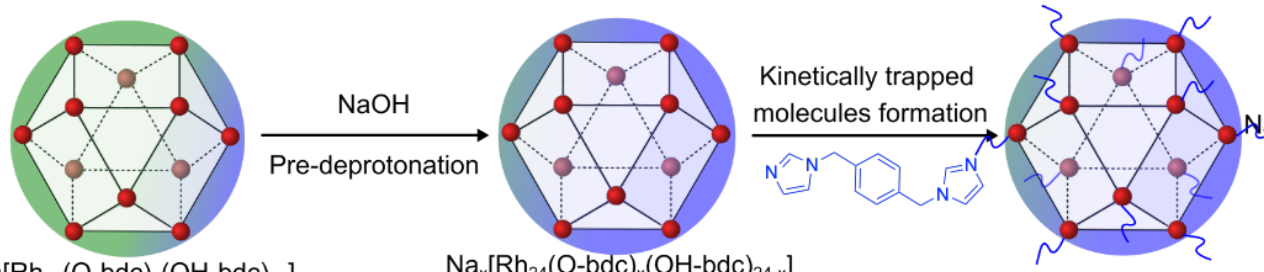

$\mathrm{Na}_{x}\left[\mathrm{Rh}_{24}(\mathrm{O}-\mathrm{bdc})_{x}(\mathrm{OH}-\mathrm{bdc})_{24-\mathrm{x}}\right](\mathrm{bix})_{12}$ $(\mathrm{x}=1,6,12,24)$

$\mathrm{Na}\left[\mathrm{Rh}_{24}(\mathrm{O}-\mathrm{bdc})_{1}(\mathrm{OH}-\mathrm{bdc})_{23}\right]$ $\mathrm{Na}_{\mathrm{x}}\left[\mathrm{Rh}_{24}(\mathrm{O}-\mathrm{bdc})_{\mathrm{x}}(\mathrm{OH}-\mathrm{bdc})_{24-\mathrm{x}}\right]$

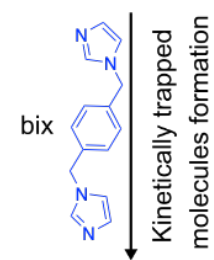
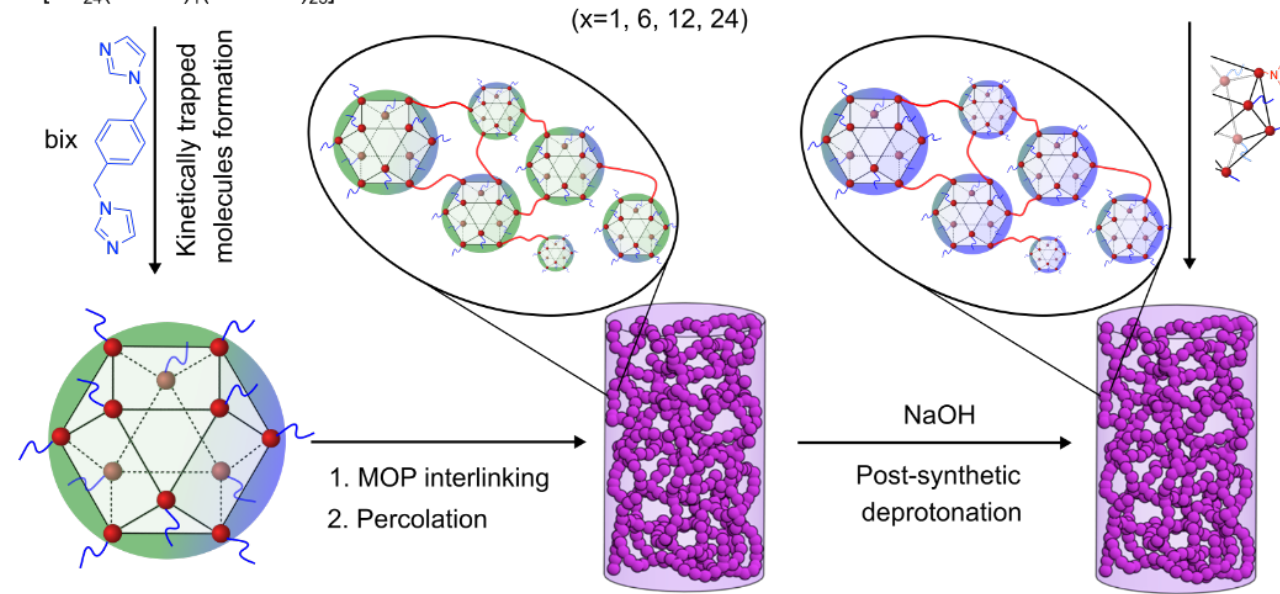

$\mathrm{Na}\left[\mathrm{Rh}_{24}(\mathrm{O}-\mathrm{bdc})_{1}(\mathrm{OH}-\mathrm{bdc})_{23}\right](\mathrm{bix})_{12}$

Gel in water/acetonitrile

Gel in water/acetonitrile
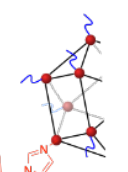

1. MOP interlinking

2. Percolation
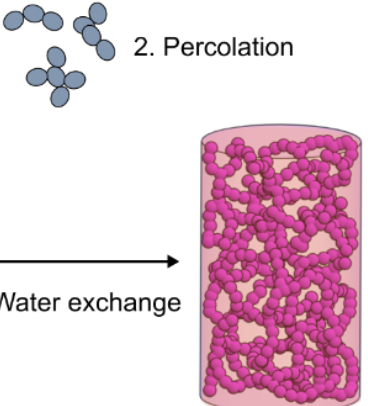

Hydrogel

Figure 1. Schematic illustration of (a) MOP with different deprotonation degree and (c) the two strategies to synthesize MOP-based hydrogel in aqueous solution

based on copper paddlewheel motifs and thus unstable in aqueous solution. Another hurdle is their poor solubility in polar solvents, further preventing the use of MOPs as gelators in the synthesis of hydrogels. ${ }^{[17]}$ To expand the potential of MOPs for further applications a strategy to develop MOP-based hydrogels is essential.

Herein, we report the synthesis of supramolecular hydrogels based on a hydrophilic MOP, $\left[\mathrm{Rh}_{2}(\mathrm{OH}-\mathrm{bdc})_{2}\right]_{12}(\mathrm{OHRhMOP} ; \mathrm{OH}-$ bdc $=5$-hydroxy-1,3-benzenedicarboxylate) with 24 hydroxyl groups $(-\mathrm{OH})$ on its periphery (Figure 1). ${ }^{[18]}$ The solubility of OHRhMOP and its kinetically trapped molecule, OHRhMOP(bix) 12 , in water is greatly increased by partial deprotonation of the MOP to yield the forms ONaRhMOP and

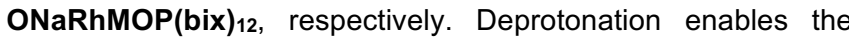
synthesis of supramolecular hydrogels despite the poor solubility of bix itself in water. We demonstrate that the deprotonation degree, which corresponds to the fraction of deprotonated $-\mathrm{OH}$ groups per MOP, plays a key role in the gel formation, the mechanical properties of resulting hydrogels and the partial decomposition of MOPs. To avoid the decomposition, an alternative route towards the hydrogels was investigated by postsynthetically tuning the deprotonation degree of MOPs within the hydrogels. Gas sorption measurements were performed on the corresponding aerogels to confirm that the cavities of the MOPs are intact inside the gels. Depending on the deprotonation degree of MOPs in the gels, their water sorption behavior can be tuned.

\section{Results and Discussion}

The isolation of kinetically trapped molecules of ONaRhMOP. Our previous studies showed that the key for successful supramolecular polymerization of MOPs is to isolate so-called kinetically trapped molecules with linkers coordinating in a monodentate fashion. In our previous investigations with $\mathbf{C}_{12}$ RhMOP and HRhMOP, ${ }^{[14 \mathrm{~b}, 19]}$ both trapped molecules were first formed with bix linkers as RhMOP(bix) ${ }_{12}$, which were isolated in DMF with good solubility. However, $\mathbf{C}_{12}$ RhMOP, HRhMOP and bix are insoluble in water. Therefore, we used the more polar MOP, OHRhMOP which has hydroxyl functionalities on its periphery, as a precursor for the polymerization in aqueous solution. We checked the solubility of OHRhMOP in water or water/organic mixing solvents and found that OHRhMOP displays good solubility in water/acetonitrile $(7: 5 \mathrm{v} / \mathrm{v})$ mixtures compared to pure water or pure acetonitrile, which does not dissolve the MOPs. This good solubility is most likely attributed to the coordination of acetonitrile molecules (MeCN) to OHRhMOP to form OHRhMOP(MeCN) $)_{12}$, which was confirmed by a color change from green to purple with a shift of maximum absorption peak in 
UV-visible spectra as discussed later (Figure S2-4). As bix is well dissolved in the same solvent mixture, this water/acetonitrile system ought to be suitable for the isolation of trapped molecules of OHRhMOP(bix) 12 .
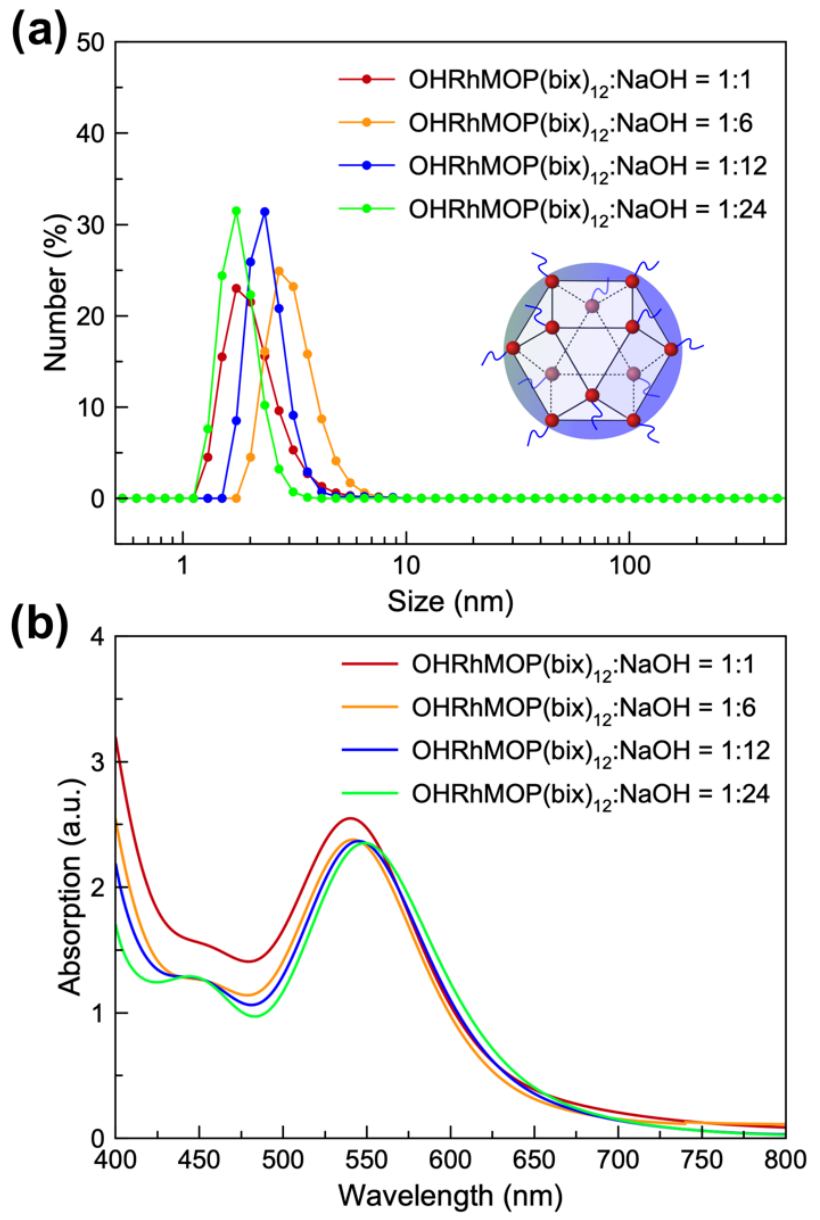

Figure 2. (a) DLS measurement of ONaRhMOP(bix) 12 in the mixing solution at different deprotonation degrees. (b) UV-vis spectra of ONaRhMOP(bix) 12 in the mixing solution at different deprotonation degrees.

A solution of 12 equivalent (eq.) of bix in water/acetonitrile was added to the solution of OHRhMOP, leading to unexpected precipitation. Analysis by ${ }^{1} \mathrm{H}$ NMR experiments confirmed the obtained solid to be the kinetically trapped molecule OHRhMOP(bix) ${ }_{12}$ (Figure S1). This suggests that the solubility of the trapped molecules was not high enough to be isolated in the mixed solution. In order to improve the solubility, the phenolic hydroxyl groups of OHRhMOP were deprotonated to yield a salt form of the MOP with sodium ions as ONaRhMOP before the addition of bix. The solution of OHRhMOP was deprotonated to different degrees by the addition of $\mathrm{NaOH}$ at different relative concentrations (1, 6, 12 or 24 eq. relative to OHRhMOP). Dynamic light scattering (DLS) measurements of these solutions after deprotonation showed a number-weighted size distribution in the range of 1-3 nm, confirming the solubility of these deprotonated MOPs (Figure S2a). After concerted addition of 12 mol eq. of bix into the basic solution of ONaRhMOP, there is no obvious change in the size distribution, which showed the presence of isolated trapped molecules of an average size of 1-3 $\mathrm{nm}$ (Figure 2a). Therefore, it was concluded that deprotonation was effective to solubilize the resulting trapped molecules, ONaRhMOP(bix) 12, in the water/acetonitrile mixture, and their solubility were not affected by the deprotonation degree.

To study the coordination environment of kinetically trapped molecules of ONaRhMOP(bix) 12, the UV-visible spectroscopy was measured before and after the addition of bix (Figure $2 b$ and $\mathrm{S} 2 \mathrm{~b}$ ). Due to the coordination of acetonitrile to the MOPs, an absorption maximum of the band $\left(\lambda_{\max }\right)$, associated with the $\pi^{*}-\sigma^{*}$ transition of the dirhodium paddlewheel moiety, was observed at $559 \mathrm{~nm}$ for the solution of ONaRhMOP (Figure S2b), which can be assigned to the chromophore of rhodium paddlewheel complex with two acetonitrile molecules coordinating on the axial sites (Figure S3-4). ${ }^{[19-20]}$ Note that this shift was independent from the deprotonation degree. After the addition of 12 eq. of bix to the ONaRhMOP solution with 1 eq. $\mathrm{NaOH}$, the $\lambda_{\max }$ was further shifted to $540 \mathrm{~nm}$ (Figure 2b). This further blue-shift is explained by the exchange of acetonitrile molecules coordinated to exohedral Rh sites of ONaRhMOPs by bix due to the higher coordinative affinity of imidazole on bix. As bix is too big to enter the pores of MOPs, the acetonitrile coordinated to internal Rh inside the MOPs remained without being exchanged by bix. This shift was confirmed with the control experiments with rhodium acetate $\left(\left[\mathrm{Rh}_{2}(\mathrm{OAc})_{4}\right]\right)$; the coordination of $1 \mathrm{eq}$. monodentate imidazole ligand, 1-dodecyl-1H-imidazole (diz), led to the same $\lambda_{\max }$ at $540 \mathrm{~nm}$ in acetonitrile (Figure S4).

Direct gelation of ONaRhMOP to form supramolecular hydrogels. To initiate the polymerization between trapped molecules, the solution ONaRhMOP(bix) 12 (0.93 mM) was heated at $60^{\circ} \mathrm{C}$ to initiate the removal of the bix molecules from the surface of ONaRhMOP and to expose the axial exohedral rhodium sites of the MOPs for the subsequent coordination with neighboring trapped ONaRhMOP(bix $)_{\mathbf{n}}$ molecules. Indeed, transparent gels were successfully obtained for all samples except for the solution with 24 eq. of $\mathrm{NaOH}$. The time-resolved DLS measurements showed the evolution of particle size as a function of time upon heating for the ONaRhMOP(bix) 12 solution with 1, 6 and 12 eq. $\mathrm{NaOH}$ (Figure 3). Once the polymerization begins, the trapped MOP molecules are hierarchically assembled into colloidal particles, leading to a steep increase in particle size. By further forming colloidal networks, the general mobility of the particles is frozen and the diffusion is stopped, rendering reliable measurement of the particle size impossible. To determine the sol-gel transition point, changes in the time-averaged scattering intensity, \langle\rangle$_{\mathrm{T}}$, were plotted as a function of time, where the time at which random fluctuations appear corresponds to the gelation point. ${ }^{[14 d]}$ The results show that, as the $\mathrm{NaOH}$ concentration increases, the gelation time is markedly delayed from 3 mins to 211 mins. Finally, when the 24 eq. of $\mathrm{NaOH}$ was added, no gelation occurs. This delay in gelation can be attributed to the electrostatic repulsion between the deprotonated MOPs. The more $-\mathrm{OH}$ groups on the MOPs are deprotonated, the more negatively charged is the resulting ONaRhMOP. This negative charge on the MOP molecules is confirmed by zeta-potential measurements (Figure S5). As a consequence, these trapped molecules are mutually repelled, thus hindering polymerization or crosslinking. For the ONaRhMOP(bix $)_{12}$ deprotonated at the highest concentration of $\mathrm{NaOH}$ (24 eq.), this electrostatic repulsion between them is too strong to allow the polymerization to occur. 

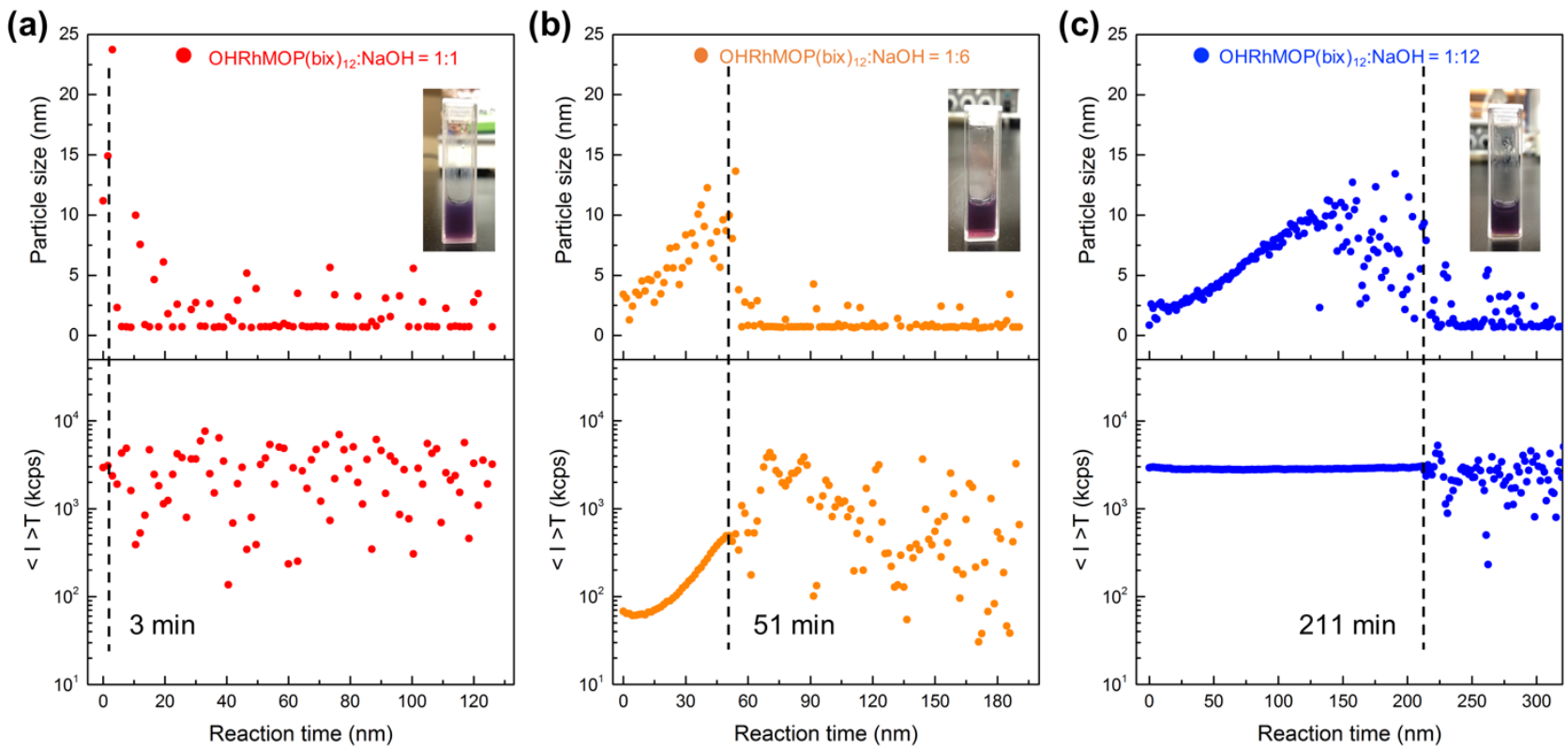

Figure 3. Time-resolved dynamic light scattering (DLS) experiments during the supramolecular polymerization of $\mathrm{ONaRhMOP(bix)})_{12}$ at $60^{\circ} \mathrm{C}$ at a concentration of $0.93 \mathrm{mM}$, showing the particle size evolution and the time-averaged scattering intensity as a function of time. Systems with different molar equivalent of $\mathrm{NaOH}$ relative to MOP were prepared: (a) 1 eq., (b) 6 eq., (c) 12 eq.

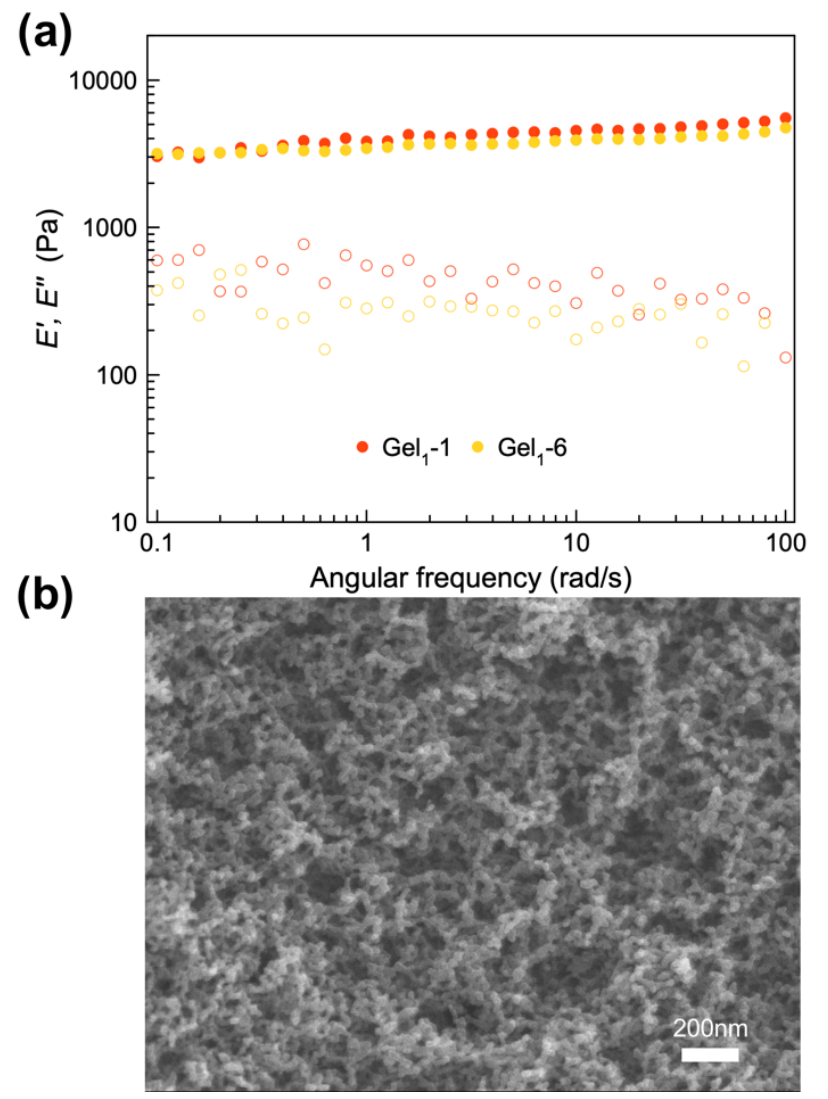

Figure 4. (a) Storage Young's modulus ( $\left.E^{\prime}\right)$ (filled circles) and loss Young's modulus (E") (hollow circles) of $\mathrm{Gel}_{1}-\mathbf{1}$ and $\mathrm{Gel}_{1}-\mathbf{6}$ versus scanning frequency (w). (b) Representative SEM image of Aerogel $\mathbf{1}_{1}-1$.
Based on the DLS results, a series of gels with various deprotonation degrees were synthesized by heating the solution of kinetically trapped ONaRhMOP(bix) 12 (see gelation conditions in Table 1). After the gelation, as-synthesized gels within the mixing solvent of water/acetonitrile were washed with fresh water/acetonitrile twice, followed by replacing the mixing solvents with distilled water for three times to yield the final hydrogel samples, named as Gel $_{1}-\mathbf{x}$ ( $\mathrm{x}$ indicates the relative molar ratio of $\mathrm{NaOH}$ added to MOP). Removal of acetonitrile from these hydrogels was confirmed by ${ }^{1} \mathrm{H}$ NMR analysis of the digested wet gel samples, showing the disappearance of the characteristic peak of acetonitrile at $2.07 \mathrm{ppm}$ (Figure S6). Rheological measurements were performed to establish the storage Young's modulus as frequency-independent $\left(E^{\prime} \approx 3.8 \mathrm{kPa}\right.$ and $3.4 \mathrm{kPa}$ for Gel $1-1$ and Gel $1-6$, respectively) and one order of magnitude higher than the loss modulus (Figure 4a). The sample of Gel $\mathbf{1}_{\mathbf{1}} \mathbf{1 2}$, however, was too fragile to be self-standing and broke upon handling (Figure S7). Therefore, it seems that the deprotonation degree of the MOPs affects not only the gelation kinetics, but also the mechanical properties of the resulting hydrogels, which becomes more fragile at higher deprotonation degree.

Similar to the previously reported organogels assembled from HRhMOP and $\mathbf{C}_{12}$ RhMOP, ${ }^{[14 b, 19]}$ the characteristic colloidal networks of all Gel $\mathbf{1}_{1} \mathbf{x}$ were revealed by the scanning electron microscopy (SEM) measurements. In the SEM images of their corresponding aerogels, Aerogel $1-\mathbf{x}$, which were prepared by replacing water in the gels with acetone for three times and then supercritical $\mathrm{CO}_{2}$ drying, similar colloidal networks were observed for all samples and the structures were not affected by the deprotonation degree (Figure $4 \mathrm{~b}$ and S8). ${ }^{1} \mathrm{H}$ NMR analysis of the acid-digested aerogels was performed to identify the ratio of bix/MOP within the $\mathrm{Gel}_{1}-\mathbf{x}$ samples (Figure S9). Contrary to our expectation, the bix/MOP ratio was estimated to be higher than 12 for all Gel $_{1}-\mathbf{x}$ samples, as listed in Table 1; the compositions 
of $\mathrm{Gel}_{1}-1, \mathrm{Gel}_{1}-\mathbf{6}$ and $\mathrm{Gel}_{1}-12$ are $\mathrm{Na}_{1}\left[\mathrm{Rh}_{24}(\mathrm{O}-\mathrm{bdc})_{1}(\mathrm{OH}-\right.$ bdc $\left.)_{23}\right](\mathbf{b i x})_{13.3}, \quad \mathrm{Na}_{6}\left[\mathrm{Rh}_{24}(\mathrm{O}-\mathrm{bdc})_{6}(\mathrm{OH}-\mathrm{bdc})_{18}\right](\mathbf{b i x})_{14.2}$ and $\mathrm{Na}_{12}\left[\mathrm{Rh}_{24}(\mathrm{O}-\mathrm{bdc})_{12}(\mathrm{OH}-\mathrm{bdc})_{12}\right](\mathbf{b i x})_{14.4}$, respectively. Considering that only 12 eq. bix was added into the MOP solution for gelation and that the crosslinking of intact cages is only possible with bix/MOP ratio lower than 12, the higher bix/MOP ratio over 12 can be attributed to the decomposition of ONaRhMOP. Despite the good stability of OHRhMOP in water, the heating process of the basic solution of isolated ONaRhMOP(bix) 12 most likely induced the decomposition of ONaRhMOP. Hydrolysis of acetonitrile molecules has been reported when heated in the presence of water and strong base like $\mathrm{NaOH}$, leading to the generation of sodium acetate and ammonia, ${ }^{[21]}$ which might cause the decomposition of MOP. It seems that the dissociated ligands of ONa-bdc were washed away during the washing process, leading to the increase of the bix/ONa-bdc ratio and thus the increase of bix/MOP ratio when the composition was estimated by ${ }^{1} \mathrm{H}$ NMR (Figure S9). Note that bix/MOP ratio continuously increased from 13.3 of Gel $1-1$ to 14.2 of Gel $\mathbf{1}_{1-12}$ with increasing the $\mathrm{NaOH}$ concentration, suggesting a serious decomposition with higher $\mathrm{NaOH}$ concentration. To further confirm the MOP decomposition, ${ }^{1} \mathrm{H}$ NMR analysis was performed to detect the free molecules in the supernatants of the washing solvent of water/acetonitrile (Figure S10). The characteristic peaks of bix and ONa-bdc were observed for all Gel $1-\mathbf{x}$ samples and the relative $\mathrm{ONa}$-bdc/bix ratio in the supernatant increased from 1.0 for the $\mathrm{Gel}_{1}-1$ to 2.4 for the $\mathrm{Gel}_{1}-\mathbf{1 2}$. Therefore, we can rationalize the increase of bix/MOP ratio in gel composition to be the decomposition of a small proportion of the MOP cages by removal of ONa-bdc linkers, which are then washed away in the washing process.

Table 1. Gelation condition and NMR composition of $\mathbf{G e l}_{1} \mathbf{- x}$ and $\mathbf{G e l}_{\mathbf{2}} \mathbf{- x}$ samples.

\begin{tabular}{|c|c|c|c|}
\hline Sample & MolmOP $_{\mathrm{MO}}$ : Mol $\mathrm{NaH}$ & Gelation condition & bix/MOP molar ratio \\
\hline Gel $_{1}-1$ & $1: 1$ & $60^{\circ} \mathrm{C}$ for $4 \mathrm{~h}$ & 13.3 \\
\hline Gel $_{1}-6$ & $1: 6$ & $60^{\circ} \mathrm{C}$ for $8 \mathrm{~h}$ & 14.2 \\
\hline Gel $_{1-12}$ & $1: 12$ & $60^{\circ} \mathrm{C}$ for $12 \mathrm{~h}$ & 14.4 \\
\hline $\mathrm{Gel}_{2}-1$ & $1: 1$ & $60^{\circ} \mathrm{C}$ for $4 \mathrm{~h}$ & 13.4 \\
\hline Gel $_{2}-6$ & $1: 6$ & $60^{\circ} \mathrm{C}$ for $4 \mathrm{~h}$ & 13.4 \\
\hline Gel$_{2}-12$ & $1: 12$ & $60^{\circ} \mathrm{C}$ for $4 \mathrm{~h}$ & 13.4 \\
\hline $\mathrm{Gel}_{2}-24$ & $1: 24$ & $60^{\circ} \mathrm{C}$ for $4 \mathrm{~h}$ & 13.1 \\
\hline
\end{tabular}

Post-synthetic deprotonation of supramolecular hydrogels. To prepare the MOP hydrogel with a high deprotonation degree but without detrimental MOP decomposition, we applied a post-synthetic deprotonation protocol after the gelation, as shown in Scheme 1. The concentration of OHRhMOP for gelation here was increased from $0.93 \mathrm{mM}$ to $1.4 \mathrm{mM}$ to guarantee the mechanical stability for the deprotonation process (Figure S11-12). To ensure the solubility of the kinetically trapped molecules, only 1 eq. $\mathrm{NaOH}$ was added for the initial deprotonation, followed by gelation in the same condition as $\mathrm{Gel}_{1}-\mathbf{1}$. The obtained gel, named $\mathrm{Gel}_{\mathbf{2}} \mathbf{- 1}$, was then soaked for 3 days in water containing different content of $\mathrm{NaOH}$ to yield hydrogels named as $\mathbf{G e l}_{2} \mathbf{- x}$ ( $\mathrm{x}$ indicates the total ratio of $\mathrm{NaOH}$ to MOP, $x=1,6,12$ and 24 here).
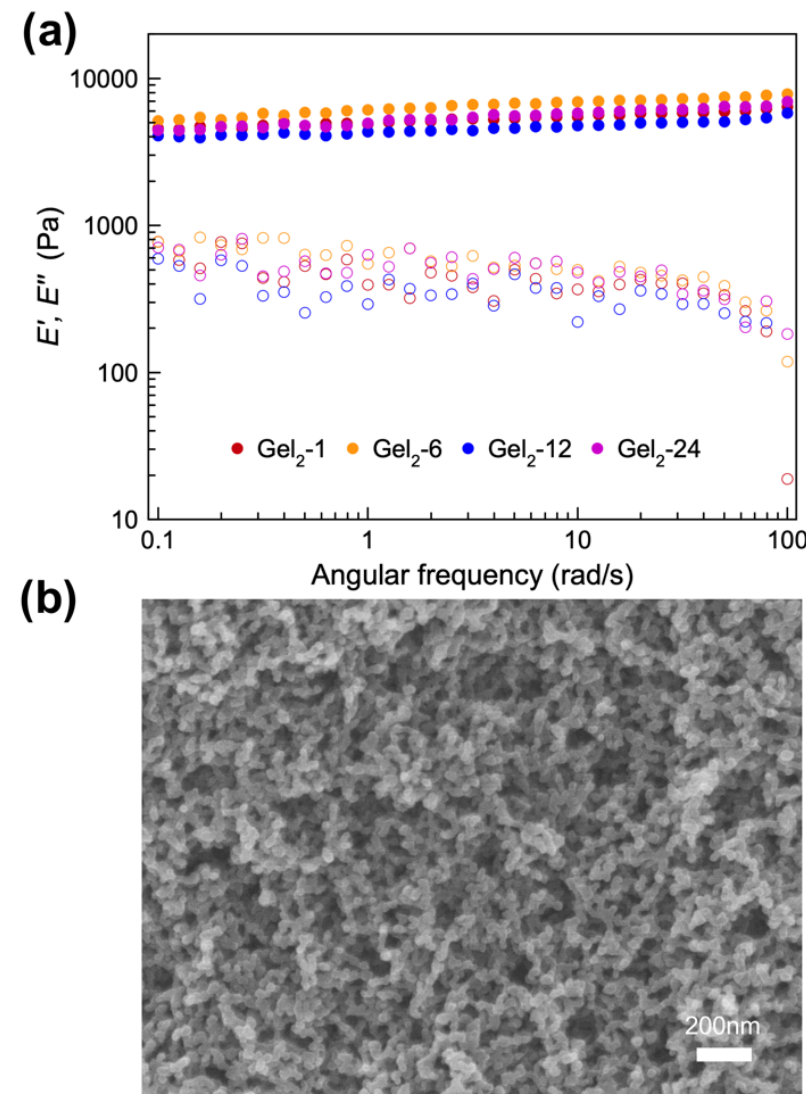

Figure 5. (a) Storage Young's modulus (E') (filled circles) and loss Young's modulus (E") (hollow circles) of $\mathbf{G e l}_{2}-\mathbf{1}, \mathbf{G e l}_{2}-\mathbf{6}, \mathbf{G e l}_{2}-\mathbf{1 2}$ and $\mathbf{G e l}_{2}-24$ versus scanning frequency $(\omega)$. (b) Representative SEM image of Aerogel $2-1$.

Deprotonation of $\mathbf{G e l}_{2}-\mathbf{x}$ samples was evaluated by measuring the $\mathrm{pH}$ of the aqueous solution used for the postsynthetic deprotonation before and after immersion of the gel (Figure S13). For all samples, the pH of the solution dropped after the deprotonation process, as listed in Table S1. The amount of $\mathrm{NaOH}$ consumed during this process increased with increasing the $\mathrm{NaOH}$ concentration, and the resulting amount of hydroxyl groups deprotonated in one MOP was calculated to be $6.3,10.9$ and $\mathbf{2 2 . 7}$ for $\mathbf{G e l}_{\mathbf{2}} \mathbf{- 6}, \mathbf{G e l}_{\mathbf{2}} \mathbf{- 1 2}$ and $\mathbf{G e l}_{\mathbf{2}} \mathbf{2 4}$, respectively. Note that the -OH groups in MOP are weakly acidic and that deprotonation here is under an equilibrium. Therefore, the calculated amount of consumed $\mathrm{NaOH}$ does not directly correspond to the true deprotonation degree. However, this result confirmed that the reaction equilibrium is successfully directed towards the deprotonation of the MOP at higher $\mathrm{NaOH}$ concentration. Because the post-synthetic deprotonation was performed at room temperature, the possible hydrolysis of acetonitrile can also be avoided unlike the former strategy where the pre-deprotonated MOP was used for gelation.

Rheology measurements revealed that all $\mathbf{G e l}_{2}-\mathbf{x}$ samples had higher mechanical stiffness than Gel $_{1}-\mathbf{x}$ (for instance, $E^{\prime} \approx 5.0$ $\mathrm{kPa}$ for $\mathrm{Gel}_{2}-1$ and $E^{\prime} \approx 3.8 \mathrm{kPa}$ for $\mathrm{Gel}_{1}-1$ ) due to the increasing concentration of MOPs used for gel formation (Figure 5a and S12), corresponding to our previous observation for the concentration- 

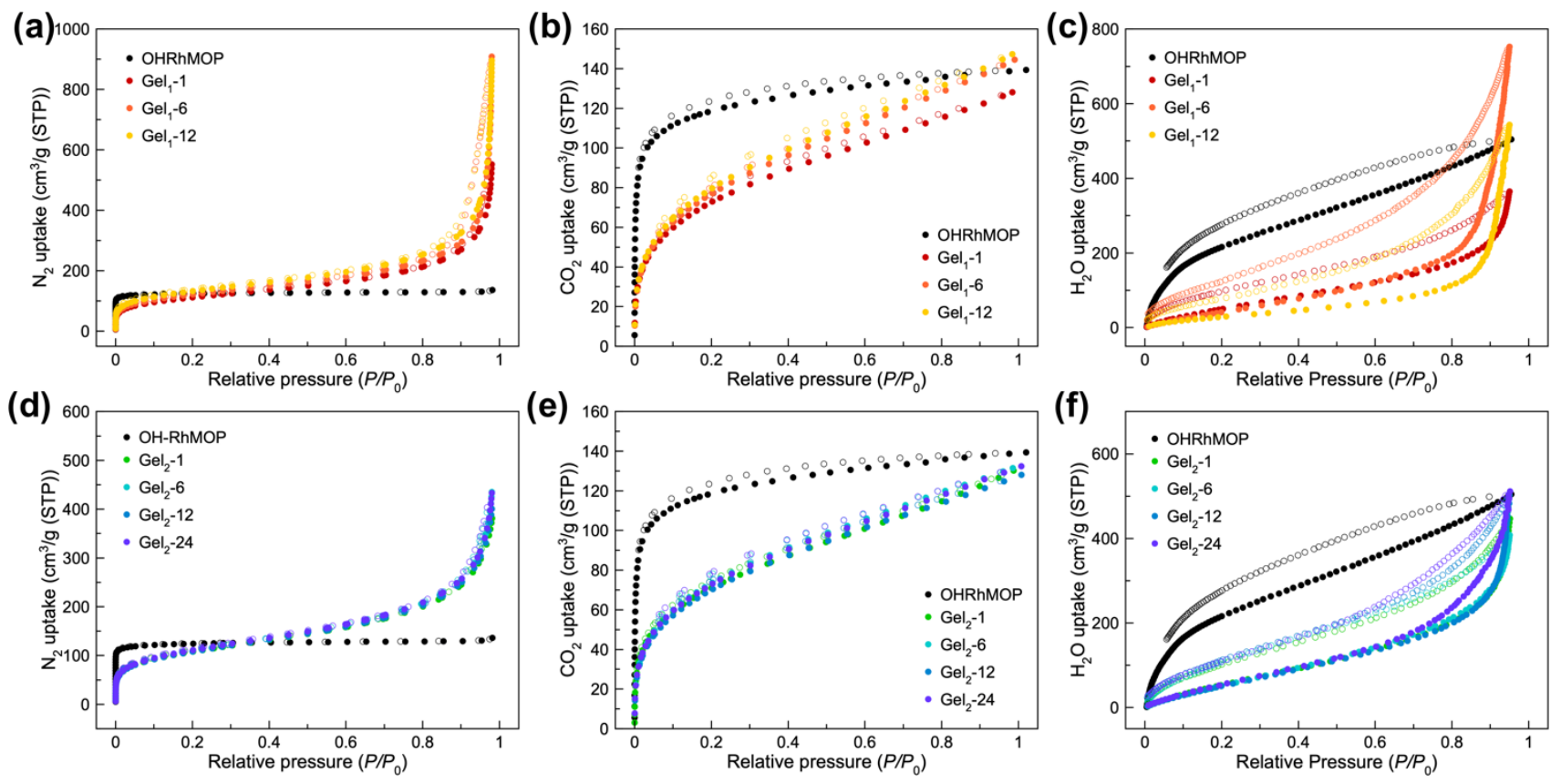

Figure 6. $\mathrm{N}_{2}$ adsorption isotherm at $77 \mathrm{~K}$ for (a) Aerogel $1_{1-x}$ samples and (d) Aerogel ${ }_{2}-\mathbf{x}$ samples. $\mathrm{CO}_{2}$ adsorption isotherm at $195 \mathrm{~K}$ for (b) Aerogel $\mathbf{1}_{1} \mathrm{x}$ samples and (e) Aerogel $\mathrm{I}_{2}-\mathrm{X}$ samples. $\mathrm{H}_{2} \mathrm{O}$ vapor isotherm at $298 \mathrm{~K}$ for (c) Aerogel ${ }_{1}-\mathrm{X}$ samples and (f) Aerogel $_{2}-\mathrm{X}$ samples.

dependent mechanical properties of $\mathbf{C}_{12}$ RhMOP gels. ${ }^{[14 \mathrm{~d}]}$ The SEM images of the corresponding aerogels showed the presence of hierarchical colloidal networks, which were not obviously damaged by the post-synthetic deprotonation process (Figure $5 \mathrm{~b}$ and S14). Table 1 listed the composition of each $\mathbf{G e l}_{2}-\mathbf{x}$ samples estimated from the ${ }^{1} \mathrm{H}$ NMR measurements of their digested aerogels (Figure S15). No obvious change of the bix/MOP ratio was observed even after the post-synthetic deprotonation. These results indicate that the post-synthetic method circumvents the serious decomposition of ONaRhMOP and maintains the mechanical stiffness of the gels at the high deprotonation degree.

Porous properties of supramolecular aerogels. In contrast to the polymer linker-based cavity-containing supramolecular gels, the use of shorter linker, bix, effectively prevents the MOP cavity to be blocked, which allows to maintain its permanent porosity within the hierarchical gel structures. Thanks to the good chemical stability of rhodium-based MOPs, the aerogels obtained here (Aerogel $\mathbf{~}_{\mathbf{1}} \mathbf{- x}$ and Aerogel $_{2}-\mathbf{x}$ ) are able to withstand the activation process at $120{ }^{\circ} \mathrm{C}$ under vacuum, allowing us to study their porosity by gas adsorption measurements. Figure $6 a$ and $6 d$ showed the $N_{2}$ sorption isotherms of Aerogel ${ }_{1}-\mathbf{x}$, Aerogel $_{2}-\mathbf{x}$ and pure OHRhMOP powders. At $77 \mathrm{~K}$, all aerogel samples displayed a sharp uptake of $\mathrm{N}_{2}$ at the low-pressure region, characteristic of microporous materials. To analyze their microporosity, the pore size distribution (PSD) was calculated by non-local density functional theory (NLDFT). As shown in Figure S17-18, the pore at $0.65 \mathrm{~nm}$ observed in OHRhMOP was maintained in all the gel samples with the pore size centered at $0.61 \mathrm{~nm}$. This indicates that, even though partial decomposition occurred, most of the MOP cavities were preserved inside the gel networks. In addition, the PSD of all aerogel samples showed the characteristic pore sizes at 1.4 and $2.8 \mathrm{~nm}$, which were most likely attributed to the external pores generated by the linkage of MOPs and bix linkers. In the higherpressure region, all aerogels showed a gradual increase of $\mathrm{N}_{2}$ uptake, while the pure OHRhMOP presented no uptake. At pressures close to $P / P_{0} \sim 1.0$, all aerogels showed a steep increase of $\mathrm{N}_{2}$ adsorption, which should be related to the $\mathrm{N}_{2}$ condensation on the surface of colloidal networks. $\mathrm{CO}_{2}$ sorption measurements $(195 \mathrm{~K})$ of all aerogel samples were also performed to show a similar sorption behavior, again demonstrating the permanent porosity of the aerogels (Figure $6 \mathrm{~b}$ and $6 \mathrm{e}$ ). For Aeroge $\mathbf{l}_{1}-\mathbf{x}$, the samples with higher deprotonation degrees seemed to show higher total uptakes of $\mathrm{N}_{2}$ and $\mathrm{CO}_{2}$. However, this difference might stem from the serious decomposition of MOPs and thus major change of the molecular composition of aerogels as discussed above. On the other hand, the post-synthetic deprotonation allowed us to circumvent the decomposition of Aerogel $\mathbf{l}_{2}-\mathbf{x}$. As shown in Figure $6 \mathrm{~d}$ and $6 \mathrm{e}$, the sorption isotherms of this gel series looked almost identical to each other, which indicates that the deprotonation degree exerts negligible influence on the sorption behavior for these gas molecules.

The hydroxyl groups on the MOP surface and their subsequent deprotonation is hydrophilic and prone to affect the sorption behavior of gels, especially for polar guest molecules. ${ }^{[22]}$ Figure $6 \mathrm{c}$ and $6 \mathrm{f}$ show the water vapor sorption isotherms of these aerogels at $298 \mathrm{~K}$. Compared with the pure OHRhMOP, all Aerogel $_{1}-\mathbf{x}$ and Aerogel $_{2}-\mathbf{x}$ samples showed lower water uptakes in the low-pressure region, indicating the weaker affinity of aerogels for water molecules due to the incorporation of hydrophobic bix linkers. In the high-pressure region, greatly increased water uptakes were observed for the aerogels, which can be attributed to the existence of mesopores and macropores inside the aerogels. Notably, the water uptake behavior of the gels at high-pressure region was obviously influenced by the 
deprotonation degree and the decomposition. Featuring similar gel structures, Aerogel $\mathbf{L}_{\mathbf{2}}-\mathbf{x}$ samples show an increased affinity toward water upon increasing the deprotonation degree, as seen in the isotherm of Aerogel $2-24$, which showed a steep water uptake at a lower pressure than the other Aerogel $_{2}-\mathbf{x}$ samples ( $\mathrm{x}$ $=1,6,12)$. For the Aerogel $_{1}-\mathbf{x}$ series, similar to the sorption isotherms of $\mathrm{N}_{2}$ and $\mathrm{CO}_{2}$, the affinity of Aerogel $1-\mathbf{x}$ toward water seems to be affected by the deprotonation and decomposition simultaneously, making it more complicated for further analysis. However, combined with the sorption results of $\mathbf{A e r o g e l}_{\mathbf{2}} \mathbf{- x}$, it shows that the deprotonation degree does influence the water sorption behavior because of the high polarity of water.

\section{Conclusion}

In conclusion, we have demonstrated two different synthetic routes towards the fabrication of porous hydrogels based on the supramolecular polymerization of OHRhMOP. The hydroxyl groups on the periphery of MOPs are able to be deprotonated by the use of base, ensuring their solubility in aqueous solution for the subsequent self-assembly with organic linker, bix. Deprotonating the OHRhMOP prior to gelation was found to play an important role in the gel formation, in which the gelation kinetics was closely related to the electrostatic repulsion between deprotonated ONaRhMOPs. Directly reacting ONaRhMOPs with linker bix yielded charged gel networks with obvious MOP decomposition, which became more serious when increasing the deprotonation degree. To circumvent the decomposition of MOP, post-synthetic deprotonation was applied after the gel formation, leading to similar hydrogel structures but with a lesser degree of decomposition. The ${ }^{1} \mathrm{H}$ NMR, SEM and rheology measurements performed to compare these two gel systems showed the mechanical properties of the gels were strongly affected by the MOP decomposition. The permanent porosity of their corresponding aerogels was confirmed by the gas sorption measurements, in which MOP deprotonation was found to influence the water sorption behavior. Further study on the decomposition mechanism and structural control of the porous MOP-based hydrogels is ongoing.

\section{Experimental Section}

\section{Materials}

Rhodium acetate ${ }^{[23]}$ and 1,4-bis(imidazol-1-ylmethyl)benzene (bix ${ }^{[14 b]}$ were synthesized according to a previously reported procedure. 5hydroxy-1,3-benzenedicarboxylic acid were purchased from SigmaAldrich and used as received. Solvents were purchased from Wako Pure Chemical Industries except those at HPLC grade were purchased from Fischer Chemicals.

\section{Synthesis of $\mathrm{Gel}_{1}-\mathrm{x}$ series hydrogels.}

OHRhMOP was dissolved in a mixture of water and acetonitrile $(7: 5 \mathrm{v} / \mathrm{v})$ to obtain a clear purple solution $(1.86 \mathrm{mM})$. Then pre-prepared solution of $\mathrm{NaOH}$ was added at different equivalent relative to OHRhMOP for deprotonation (1, 6 and $12 \mathrm{~mol}$. eq., respectively). After sonication, this purple solution was added to the water/acetonitrile solution of bix (22.32 $\mathrm{mM}, 12 \mathrm{~mol}$. eq.) under vigorous stirring. The obtained clear purple solution was then placed into a preheated oven at $60^{\circ} \mathrm{C}$ to induce gelation. According to the deprotonation degree of OHRhMOP, the heating time is set differently depending on the DLS results ( $4 \mathrm{~h}, 8 \mathrm{~h}$ and $12 \mathrm{~h}$, respectively). After gelation, the gel sample was soaked within fresh water/acetonitrile solution twice to remove the residual chemicals. Then the washed gel was immersed in distilled water for 3 days, replacing the solvent with fresh water each day to remove the acetonitrile to obtain a pure hydrogel based on OHRhMOP. The hydrogel obtained here was referred to $\mathrm{Gel}_{\mathbf{1}} \mathbf{- 1}, \mathbf{G e l}_{\mathbf{1}}$ 6, Gel $_{1}-12$ depending on the equivalent of the $\mathrm{NaOH}$ added.

\section{Synthesis of $\mathrm{Gel}_{2}-\mathrm{x}$ series hydrogels.}

OHRhMOP was dissolved in a mixture of water and acetonitrile $(7: 5 \mathrm{v} / \mathrm{v})$ to obtain a clear purple solution $(2.8 \mathrm{mM})$. Then pre-prepared solution of $\mathrm{NaOH}$ (1mol. eq. relative to OHRhMOP) was added for initial deprotonation. After sonication, this purple solution was added to the water/acetonitrile solution of bix $(33.6 \mathrm{mM}, 12 \mathrm{~mol}$. eq.) under vigorous stirring. The obtained clear purple solution was then placed into a preheated oven at $60^{\circ} \mathrm{C}$ for $4 \mathrm{~h}$ towards gelation. After gelation, the gel sample was soaked within fresh water/acetonitrile solution twice to remove the residual chemicals. Then the washed gel was immersed in distilled water for 3 days, replacing the solvent with fresh water each day to remove acetonitrile. For post-synthetic deprotonation, the obtained hydrogel was immersed into the solution of $\mathrm{NaOH}(1,6,12,24$ mol. eq., respectively) and left for two days. After reaction, the hydrogel was washed by fresh water again to get $\mathrm{Gel}_{\mathbf{2}}-\mathbf{1}, \mathrm{Gel}_{\mathbf{2}}-\mathbf{6}, \mathrm{Gel}_{\mathbf{2}}-\mathbf{1 2}, \mathrm{Gel}_{\mathbf{2}}-\mathbf{2} \mathbf{4}$ depending on the equivalent of the $\mathrm{NaOH}$ used.

\section{Synthesis of $\mathrm{Gel}_{1}-\mathrm{x}$ and $\mathrm{Gel}_{2}-\mathrm{x}$ series aerogels.}

To obtain aerogel, as-made gel was soaked with acetone for three days, with the acetone replaced each day. Then the solvent-exchanged samples were then dried by supercritical $\mathrm{CO}_{2}$ at $14 \mathrm{MPa}$ and $40^{\circ} \mathrm{C}$ for 90 mins to obtain the aerogel. Prior to sorption measurements, the aerogel sample was activated at $120^{\circ} \mathrm{C}$ under vacuum for $12 \mathrm{~h}$.

\section{Characterizations}

Thermogravimetric analyses (TGA) were performed in the temperature range from room temperature to $500^{\circ} \mathrm{C}$ at a heating rate of $10^{\circ} \mathrm{C} / \mathrm{min}$ with a Rigaku Thermo plus EVO2, under a nitrogen atmosphere. The supercritical $\mathrm{CO}_{2}$ drying process was carried out on SCLEAD-2BD autoclave (KISCO) using super-critical $\mathrm{CO}_{2}$ at $14 \mathrm{MPa}$ and $40^{\circ} \mathrm{C}$. Infrared (IR) spectroscopy data were recorded neat using a Jasco FT/IR-6100 with 1 $\mathrm{cm}^{-1}$ resolution and an accumulation of 128 scans. UV-vis was measured in a V-670 spectrophotometer (JASCO). Samples were observed using a field-emission scanning electron microscope with a JEOL Model JSM$7001 \mathrm{~F} 4$ system. Linear dynamic mechanical analysis was performed using RSA-G2 (TA instruments) by compression mode. ${ }^{1} \mathrm{H}$ NMR spectra were recorded on a Bruker Biospin DRX-600 $(600 \mathrm{MHz})$ spectrometer. For ${ }^{1} \mathrm{H}-$ NMR analysis, 5-10 mg of aerogel samples were digested in a mixture of DMSO- $_{6}(750 \mu \mathrm{l})$ and $\mathrm{DCl}(50 \mu \mathrm{l})$. The mixture was then heated at $100^{\circ} \mathrm{C}$ overnight to obtain a yellow solution. Dynamic Light Scattering (DLS) measurements and zeta potential of the MOP solutions were performed using a Zetasizer Nano ZS.

\section{Acknowledgements}

Z. W. acknowledges the China Scholarship Council (CSC) for the scholarship support. This study was supported by JSPS KAKENHI Grant Number 20K15366 (Wakate) for A. L. and 19H04575 (Coordination Asymmetry) and 18H01995 (Kiban B) for S.F. The authors thank the iCeMS Analysis Center for access to analytical instruments. 
Keywords: Cage compounds • Gels • Microporous materials • Self-assembly $\bullet$ Supramolecular chemistry

[1] J. W. Steed, Chem. Commun. 2011, 47, 1379-1383.

[2] a) M.-O. M. Piepenbrock, G. O. Lloyd, N. Clarke, J. W. Steed, Chem Rev. 2010, 110, 1960-2004; b) R. Eelkema, A. Pich, Adv. Mater. 2020 32, 1906012; c) N. M. Sangeetha, U. Maitra, Chem. Soc. Rev. 2005, 34 , 821-836.

[3] a) J. A. Foster, J. W. Steed, Angew. Chem. Int. Ed. 2010, 49, 6718-6724; b) X. Yan, T. R. Cook, J. B. Pollock, P. Wei, Y. Zhang, Y. Yu, F. Huang, P. J. Stang, J. Am. Chem. Soc. 2014, 136, 4460-4463; c) Z. Qi, C. A. Schalley, Acc. Chem. Res. 2014, 47, 2222-2233.

[4] a) X. Ji, R.-T. Wu, L. Long, C. Guo, N. M. Khashab, F. Huang, J. L. Sessler, J. Am. Chem. Soc. 2018, 140, 2777-2780; b) S. M. Mantooth, B. G. Munoz-Robles, M. J. Webber, Macromol. Biosci. 2019, 19, 1800281; c) X. Ji, H. Wang, H. Wang, T. Zhao, Z. A. Page, N. M. Khashab, J. L. Sessler, Angew. Chem. Int. Ed. 2020, 59, 23402-23412;

[5] I. Hwang, W. S. Jeon, H. J. Kim, D. Kim, H. Kim, N. Selvapalam, N. Fujita, S. Shinkai, K. Kim, Angew. Chem. Int. Ed. 2007, 119, 214-217.

[6] W. Deng, H. Yamaguchi, Y. Takashima, A. Harada, Angew. Chem. Int. Ed. 2007, 46, 5144-5147.

[7] D.-S. Guo, Y. Liu, Chem. Soc. Rev. 2012, 41, 5907-5921.

[8] T. Ogoshi, T. Kakuta, T.-a. Yamagishi, Angew. Chem. Int. Ed. 2019, 58, 2197-2206.

[9] a) S.-L. Huang, G.-X. Jin, H.-K. Luo, T. S. A. Hor, Chem. Asian. J. 2015 10, 24-42; b) B. Li, B. Wang, X. Huang, L. Dai, L. Cui, J. Li, X. Jia, C. Li, Angew. Chem. Int. Ed. 2019, 58, 3885-3889.

[10] a) M. Fujita, M. Tominaga, A. Hori, B. Therrien, Acc. Chem. Res. 2005, 38, 369-378; b) J. R. Nitschke, Acc. Chem. Res. 2007, 40, 103-112; c) R. Chakrabarty, P. S. Mukherjee, P. J. Stang, Chem. Rev. 2011, 111, 6810-6918; d) H. Sepehrpour, W. Fu, Y. Sun, P. J. Stang, J. Am. Chem. Soc. 2019, 141, 14005-14020.

[11] a) D. J. Tranchemontagne, Z. Ni, M. O'Keeffe, O. M. Yaghi, Angew. Chem. Int. Ed. 2008, 47, 5136-5147; b) N. Hosono, S. Kitagawa, Acc. Chem. Res. 2018, 51, 2437-2446; c) Y. Sun, C. Chen, P. J. Stang, Acc. Chem. Res. 2019, 52, 802-817; d) X. Hu, S. Feng, J. Du, L. Shao, J. Lang, C. Zhang, S. P. Kelley, J. Lin, S. J. Dalgarno, D. A. Atwood, J. L. Atwood, Chem. Sci. 2020, 11, 12547-12552.

[12] a) J.-R. Li, D. J. Timmons, H.-C. Zhou, J. Am. Chem. Soc. 2009, 131 6368-6369; b) G. Lal, M. Derakhshandeh, F. Akhtar, D. M. Spasyuk, J.B. Lin, M. Trifkovic, G. K. H. Shimizu, J. Am. Chem. Soc. 2019, 141, 1045-1053; c) Z. Niu, S. Fang, X. Liu, J.-G. Ma, S. Ma, P. Cheng, J. Am. Chem. Soc. 2015, 137, 14873-14876; d) A. V. Zhukhovitskiy, M. Zhong, E. G. Keeler, V. K. Michaelis, J. E. P. Sun, M. J. A. Hore, D. J. Pochan, R. G. Griffin, A. P. Willard, J. A. Johnson, Nat. Chem. 2016, 8, 33-41; e) J. Lee, J. H. Kwak, W. Choe, Nat. Commun. 2017, 8, 14070; f) M. A. Andrés, A. Carné-Sánchez, J. Sánchez-Laínez, O. Roubeau, J. Coronas, D. Maspoch, I. Gascón, Chem. Eur. J. 2020, 26, 143-147.

[13] a) Y. Gu, E. A. Alt, H. Wang, X. Li, A. P. Willard, J. A. Johnson, Nature 2018, 560, 65-69; b) N. J. Oldenhuis, K. P. Qin, S. Wang, H.-Z. Ye, E. A Alt, A. P. Willard, T. Van Voorhis, S. L. Craig, J. A. Johnson, Angew. Chem. Int. Ed. 2020, 59, 2784-2792; c) L. Shao, B. Hua, X. Hu, D. Stalla S. P. Kelley, J. L. Atwood, J. Am. Chem. Soc. 2020, 142, 7270-7275; d) J. Uchida, M. Yoshio, S. Sato, H. Yokoyama, M. Fujita, T. Kato, Angew. Chem. Int. Ed. 2017, 56, 14085-14089; e) L. Li, S. Xiang, S. Cao, J. Zhang, G. Ouyang, L. Chen, C.-Y. Su, Nat. Commun. 2013, 4, 1774.

[14] a) S. Furukawa, N. Horike, M. Kondo, Y. Hijikata, A. Carné-Sánchez, P. Larpent, N. Louvain, S. Diring, H. Sato, R. Matsuda, R. Kawano, S. Kitagawa, Inorg. Chem. 2016, 55, 10843-10846; b) A. Carné-Sánchez, G. A. Craig, P. Larpent, T. Hirose, M. Higuchi, S. Kitagawa, K. Matsuda, K. Urayama, S. Furukawa, Nat. commun. 2018, 9, 1-8; c) A. CarnéSánchez, G. A. Craig, P. Larpent, V. Guillerm, K. Urayama, D. Maspoch, S. Furukawa, Angew. Chem. Int. Ed. 2019, 58, 6347-6350; d) A. Legrand, G. A. Craig, M. Bonneau, S. Minami, K. Urayama, S. Furukawa, Chem. Sci. 2019, 10, 10833-10842.

[15] a) S. Ganta, D. K. Chand, Inorg. Chem. 2018, 57, 3634-3645; b) C. Lu, M. Zhang, D. Tang, X. Yan, Z. Zhang, Z. Zhou, B. Song, H. Wang, X. Li, S. Yin, H. Sepehrpour, P. J. Stang, J. Am. Chem. Soc. 2018, 140, 7674-
7680; c) P. Sutar, V. M. Suresh, K. Jayaramulu, A. Hazra, T. K. Maji, Nat. Commun. 2018, 9, 3587; d) S.-C. Wei, M. Pan, Y.-Z. Fan, H. Liu, J. Zhang, C.-Y. Su, Chem. Eur. J. 2015, 21, 7418-7427.

[16] a) J. A. Foster, R. M. Parker, A. M. Belenguer, N. Kishi, S. Sutton, C. Abell, J. R. Nitschke, J. Am. Chem. Soc. 2015, 137, 9722-9729; b) Y. Qin, L.-L. Chen, W. Pu, P. Liu, S.-X. Liu, Y. Li, X.-L. Liu, Z.-X. Lu, L.-Y. Zheng, Q.-E. Cao, Chem. Commun. 2019, 55, 2206-2209; c) P. Howlader, P. S. Mukherjee, Chem. Sci. 2016, 7, 5893-5899.

[17] a) G. Liu, Y. Di Yuan, J. Wang, Y. Cheng, S. B. Peh, Y. Wang, Y. Qian, J. Dong, D. Yuan, D. Zhao, J. Am. Chem. Soc. 2018, 140, 6231-6234; b) S. Mollick, S. Mukherjee, D. Kim, Z. Qiao, A. V. Desai, R. Saha, Y. D. More, J. Jiang, M. S. Lah, S. K. Ghosh, Angew. Chem. Int. Ed. 2019, 58, 1041-1045; c) S. Mollick, S. Fajal, S. Mukherjee, S. K. Ghosh, Chem. Asian. J. 2019, 14, 3096-3108; d) E. G. Percástegui, T. K. Ronson, J. R. Nitschke, Chem. Rev. 2020, 120, 13480-13544.

[18] a) A. Carné-Sánchez, J. Albalad, T. Grancha, I. Imaz, J. Juanhuix, P. Larpent, S. Furukawa, D. Maspoch, J. Am. Chem. Soc. 2019, 141, 40944102; b) T. Grancha, A. Carné-Sánchez, L. Hernández-López, J. Albalad, I. Imaz, J. Juanhuix, D. Maspoch, J. Am. Chem. Soc. 2019, 141, 1834918355.

[19] A. Carné - Sánchez, G. A. Craig, P. Larpent, V. Guillerm, K. Urayama, D. Maspoch, S. Furukawa, Angew. Chem. Int. Ed. 2019, 131, 6413-6416.

[20] E. Warzecha, T. C. Berto, C. C. Wilkinson, J. F. Berry, J. Chem. Edu. 2019, 96, 571-576.

[21] Z. Wang, S. M. Richter, M. J. Rozema, A. Schellinger, K. Smith, J. G. Napolitano, Org. Process Res. Dev. 2017, 21, 1501-1508.

[22] a) L. Kong, R. Zou, W. Bi, R. Zhong, W. Mu, J. Liu, R. P. S. Han, R. Zou, J. Mater. Chem. A 2014, 2, 17771-17778; b) R. Tan, A. Wang, R. Malpass-Evans, R. Williams, E. W. Zhao, T. Liu, C. Ye, X. Zhou, B. P. Darwich, Z. Fan, L. Turcani, E. Jackson, L. Chen, S. Y. Chong, T. Li, K. E. Jelfs, A. I. Cooper, N. P. Brandon, C. P. Grey, N. B. McKeown, Q. Song, Nat. Mater. 2020, 19, 195-202; c) L. Stegbauer, M. W. Hahn, A. Jentys, G. Savasci, C. Ochsenfeld, J. A. Lercher, B. V. Lotsch, Chem. Mater. 2015, 27, 7874-7881.

[23] S. Furukawa, N. Horike, M. Kondo, Y. Hijikata, A. Carné-Sánchez, P Larpent, N. Louvain, S. p. Diring, H. Sato, R. Matsuda, Inorg. Chem. 2016, 55, 10843-10846. 